\title{
A motion-planning method for dexterous hand operating a tool based on bionic analysis
}

\author{
Bo Wei, Congying Deng* , Jie Zhao and Yi Zhang \\ Chongqing University of Posts and Telecommunications, Chongqing, China
}

\begin{abstract}
In order to meet the needs of robot's operating tools for different types and sizes, the dexterous hand is studied by many scientific research institutions. However, the large number of joints in a dexterous hand leads to the difficulty of motion planning. Aiming at this problem, this paper proposes a planning method abased on BPNN inspired by human hands. Firstly, this paper analyses the structure and function of the human hand and summarizes its typical strategy of operation. Secondly, based on the manual operation strategy, the tools are classified according to the shape and the operation mode of the dexterous hand is presented. Thirdly, the BPNN is used to train the humanoid operation, and then output the operation plan. Finally, the simulating experiments of grasping simple tools and operating complex tools are made by MATLAB and ADAMS. The simulation verifies the effectiveness of this method.
\end{abstract}

\section{Introduction}

As one of the greatest human inventions in the 20th century, robots are widely used in many fields to replace or assist the human on work. To operate the complex labour tools, robots need a dexterous hand to accomplish so many kinds of tasks. The traditional end-effector of robot is too simple to operate kinds of tools. However, the dexterous hand with multiple joints has to face the problems of complex Motion planning. Therefore, this paper presents a planning strategy based on BP Neural Network which enables the dexterous hand to use a wide range of tools like human and complete the operation task.

In recent decades, a large number of dexterous hands are invited and some of them have been applied in the fields of military and outer space[1,2,3]. In 2010, Aaron $\mathrm{m}$. Dollar and Robert d. Howe proposed a very simple manipulator design concept[4]. Although the manipulator can't complete the delicate operation, this design concept showed a manipulator' s high adaptability to operate[5].Engineers from the German Space Center have developed the DLR Hand Arm System based on the full analysis of the human body's physiological structure. This hand is very flexible, but it also brings 38 motors and complex kinematic[6]. The robot astronaut Robonaut 2, developed by NASA, has a dexterous hand with 12 Dofs[7,8]. Shadow Company began to develop a five-finger hand with 24 joints in $2004[9,10]$.

In this paper, the motion planning of multi-degree-offreedom manipulative dexterous hand is studied. First of all, the bionic system of the human hand is analyzed, including structure and function. Then, six classic grasping methods are summarized. Secondly, the operation mode of human hand is classified, and it is classified according to the shape of different tools. Thirdly, based on classification, BP neural network is used to plan the motion of dexterous hand. Finally, the simulation experiment proves the correctness of the operation plan.

\section{BIONICS OF HUMAN HANDS}

Nowadays, in the multi-fingered hand research, there is a contradiction between the mechanical configurations and the operating function. The contradiction can be described as if the dexterous hand is simple, its reliability is high relatively. But the hand will not complete accurate operation mission - and vice versa. After thousands of years of human evolution, human hands have found the balance between mechanical configurations and operating function.

Based on this, we analysed both the topological structure and function of a man's hand, and extract the characteristic of bionics significance, such as the functional analysis of a man's hand, coupling finger gestures with hands and finger strength and flexibility analysis. Through the analysis of hand function, this paper uses BPNN(back propagation neural network) to make the motion planning for dextrous hand. This method could improve the operation ability, and at the same time, the difficulty of control is reduced.

\subsection{Structure of human hand}

The structure of the hand is very complex. With the development of anatomy, modern medical research on human hand's skeleton structure has been perfected.

\footnotetext{
* Corresponding author: bowei@cqupt.edu.cn
} 
A human hand consists of the thumb, forefinger, middle finger, ring finger and little finger, as well as a palm. That consists of 44 tendons and 27 bones. The skeletal structure is shown in Fig.1.

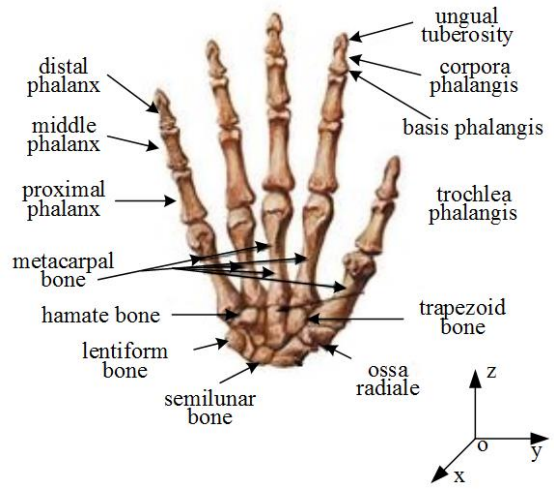

Fig.1. Skeletal structure of human hand

As is seen in Fig.1, three orthogonal axes are defined to describe the motion trajectory. The $\mathrm{x}$ axis is perpendicular to the ground and the direction is from top to bottom. The $\mathrm{Y}$-axis is parallel to the ground, and the direction is horizontal extension. The $\mathrm{z}$ axis is parallel to the ground and the direction is from front to back. Motions of the hand are as below.

a) Internal Rotation and Outward Rotation. That joint rotats long the $\mathrm{X}$-axis. If the joint roates towards the inner circle, it's called the inner rotation. If the joint roates towards the outer circle, it's called the outer rotation.

b) Flexion and Extension. That joint rotats long the $\mathrm{Y}$-axis. If the angle between the two joints becomes smaller, it's called flexion. If the angle between the two joints becomes larger, it's called extension.

c) Inward and Outward Extending. that joint rotats long the Z-axis. If the joint rotats toward the origin point, it's called inward extending. The opposite is called outward extending.

d) Circular motion. The joint is rotated along the axial direction.

The base joint of human hand's finger has two degrees of freedom in two directions, which makes the griping more stable and the operation more dexterous.

When the operating target is so large that it cannot be fully enveloped by palm(Fig.2(a)), each finger moves sideways and the palm opens to increase the covering area of the hand to achieve a more reliable grip. When the operation is required to be precise, such as playing a guitar(Fig.2(b)), the four fingers can make sure the hand to complete the precise operation.

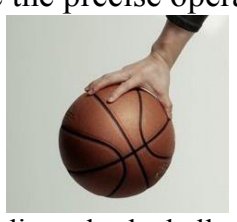

(a) holding a basketbal

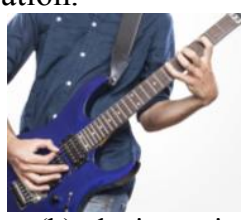

(b) playing guitar
Fig.2. Motion ability of human fingers

\subsection{Function of Human Hand}

Even though the sizes and shapes of different human hands are different, the hands have similar functions.
This is mainly due to the very reasonable structure and proportion of the hands. As can be seen in Fig.3, the shapes of two hands are different, but they have the same degree of freedom configuration and the proportions of each part are roughly the same. As a result, for the same object, the two hands operate roughly the same way[12].

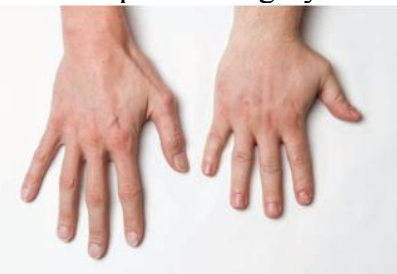

Fig.3. The sizes of different don's influence the operating function

In the study of human hand anatomy, there are many kinds of opration mode classification. The hand opration modes can be divided into six types, as shown in Fig.4.

a) Strong grasp. The thumb is relative to the other four fingers and is completely bent, such as holding a tool.

b) Pinching. The thumb and forefinger touch each other together, such as pinching a needle.

c) Lift. The four fingers, beside the thumb, are used to lift a target, such as a bag.

d) Tripod pinc. The index finger, thumb and middle finger are combined to opearte somthing, such as pen.

e) Sphere grasp. All five fingers bend and grasp, such as grasp a ball.

f) Lateral pinch. A side of the index finger is opposite to the inside of the thumb, such as pinching a key.

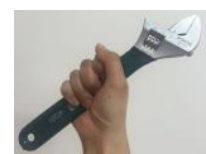

(a)

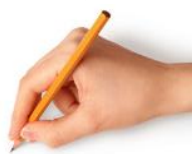

(d)

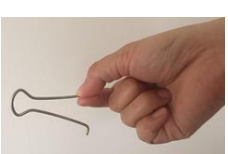

(b)

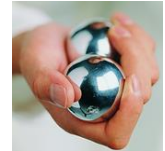

(e)

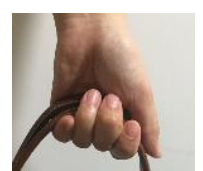

(c)

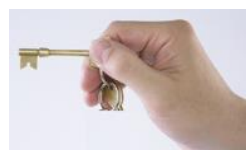

(f)
Fig.4. Six operating modes of hunman hand

\section{Operation strategy of dextrous hand}

For different tools, the way dextrous hand operates is different. And for the same kind of tool, because of the tool size and its open-close state, the operating position and the fingers' degrees of motion are different. In the previous section, the operation modes of human hand has been analysed. In this section, the operation mode of dextrous hand is judged by BP neural network and the joint angles of each joint are also given by it.

\subsection{Operation mode analysis of Dexterous hand}

After analyzing the function of human hand, the operation methods vary from tool to tool. Therefore, in order to satisfy the operation demand of different kinds 
of tools, the operating methods of tools in different shapes are classified in Fig.5.

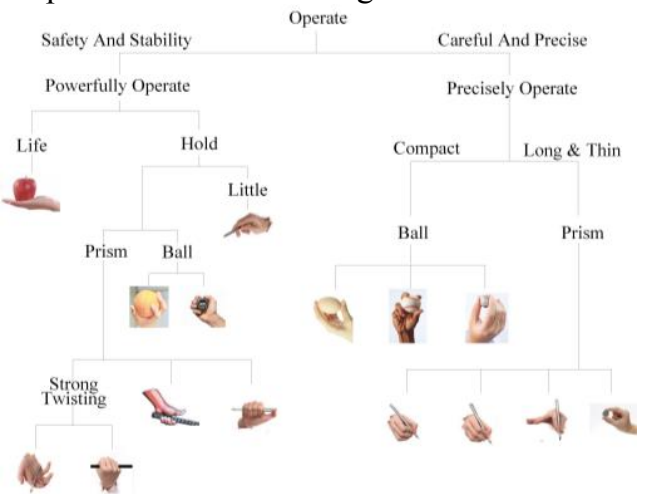

Fig.5. Operating methods of tools in different shapes

\subsection{Motion planning based on BP neural network}

The way how human hands operate a tool mainly depends on its shape, size and current state. Mostly, the operation mode is based on knowledge base. However, it takes a long process to build a knowledge base and it is difficult to guarantee the integrity of the knowledge base. The artificial network has the ability to learn rapidly, complete the complex nonlinear mapping and deduce the very approximate conclusions from incomplete input. Therefore, inspired by the way human hands operation, the artificial neural network is used to make the motion plan of dexterous hand. And because BP neural network has good self-learning ability, generalization ability and fault tolerance, the BP neural network(BPNN) is selected to complete the motion planning.

A pattern recognition system mainly includes training process and identification process. The training process is mainly based on a large number of samples and a designed classifier. The identification is the process of matching unknown patterns with trained classifiers. The core of BPNN the correction of the network weight value and the threshold value, so that the error function decreases along the gradient direction.

In this paper, we selected a three-layer BPNN for identification. As can be seen in Fig.6, the tools are divided into three categories: gun style tool, clamp style tool and stick style tool. The transformation function of implicit layer is generally nonlinear function, such as stype function or hyperbolic tangent function. The transformation function of the output layer can be nonlinear or linear, which is determined by the need for input and output mapping relationships.

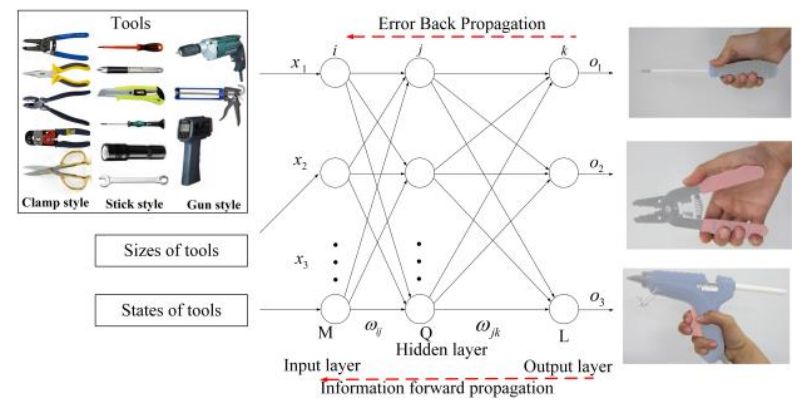

Fig.6. BPNN for motion planning

\subsection{BPNN for dextrous hand operation}

According to the task requirements of the dexterous hand operation tool, the neural network structure is set as follows. The input layer has $\mathrm{M}$ nodes. The hidden layer has Q nodes, which are the connection weights between the input layer and hidden layer. The output layer has L nodes, which are the connection weights between the hidden layer and output layer. The input values of the hidden layer and output layer are the weighted sum of the former layer. The degree of excitation of each node is determined by its excitation function. The Constant distance characteristics are put in the network and the sampling training is carried, then, sample full training is carried.

If $\mathrm{E}<0.01$ for 5 consecutive times, the training is finished. If the training times are over 5000 times, the training is failure. In order to increase the robustness of the system, the output of the BPNN is the grade of membership of a tool. If the maximum output is smaller than 0.6 , that means the target is not belonging any style. If there are several maximum outputs, that means the judge is wrong.

\section{Eexperiments and Results}

\subsection{Parameters of BPNN}

\subsubsection{Input parameters}

The tools are classified as 3 types. The type is defined as $\mathrm{t}(\mathrm{t}=1,2,3) . \mathrm{t}=1$ means clamps style, $\mathrm{t}=2$ means stick style, $\mathrm{t}=3$ means gun style.

Classification of tools, tool type $t(t=1,2,3)$ as an input to BP neural network.

Even though the tools are the same type, they may have different sizes. Therefore, the tool size is also an input to the neural network. The tool size is set as the minimum envelope radius $\mathrm{R}$ in the plane of the tool.

Besides, the open and closed state of the clamping tool also affects the angles of finger joints. So, the open and closed state is also the input of the neural network.

In addition, the open and closed state of the clamping tool also affects the Angle of finger grasping, so it is also the input of the neural network.

\subsubsection{Output parameters}

The output of the neural network is defined as the position of the fingertip relatively to the wrist. Then the angles of the finger joints are calculated through inverse kinematics.

\subsubsection{Setting of network structure}

The input layer includes the tool type, open-close state and the minimum envelope circle radius. So the node number of input layer $\mathrm{m}$ is 3 . 
The node number of the output layer is related to the structure of the dexterous hand. In order to simplify the neural network, the output is set depending on the operation gesture. There are 15 kinds of gesture types in Fig.5. Adding the open-close state, the node number of output layer $n$ is 16

The hidden number of the output layer is determined by empirical function $s=\sqrt{m+n}+a$ or $s=\sqrt{0.43 m n+0.12 n^{2}+2.54 m+0.77 n+0.35}+0.51$, where a is an integer between 1 and 10. According to experience, the number of hidden layer nodes is 10 .

The transfer function between the input layer and the hidden layer is a kind of log-sigmoid function. The transfer function between the hidden layer and the output layer is a linear function.

\subsubsection{Network value adjustment}

In the iterative process, the change of the error function is used to adjust $\eta$.

$$
\eta(t)=\left\{\begin{array}{l}
1.2 \eta(t-1) \cdots E(n)<E(n-1) \\
0.8 \eta(t-1) \cdots E(n)<1.05 E(n-1) \\
1.2 \eta(t-1) \cdots E(n-1)<E(n) 1.05 E(n-1)
\end{array}\right.
$$

In order to ensure that each learning can be effectively corrected and accelerate the convergence process, the output of log-sigmoid is limited.

$$
f(x)=\left\{\begin{array}{l}
0.01 \cdots f(x)<0.01 \\
0.99 \cdots f(x)>0.99 \\
f(x) \cdots 0.01<f(x)<0.99
\end{array}\right.
$$

The parameters are initialized as:

$$
\begin{aligned}
\eta & =\frac{1}{\sqrt{s}} \\
\alpha & =0.8
\end{aligned}
$$

\subsection{Simulation results}

MATLAB and ADAMS are used to simulate dexterous hand to operate tools. As can be seen in Fig.6, firstly, tools and dexterous hand are established in the ADAMS. Secondly, the shape, size and the open-close state are input to MATLAB. Thirdly, the positions between the fingertips and the wrist are deduced from BPNN. Fourthly, the angles of each finger's joints are calculated by inverse kinematics. Finally, the angles are input to ADAMS and the hand is motioned.

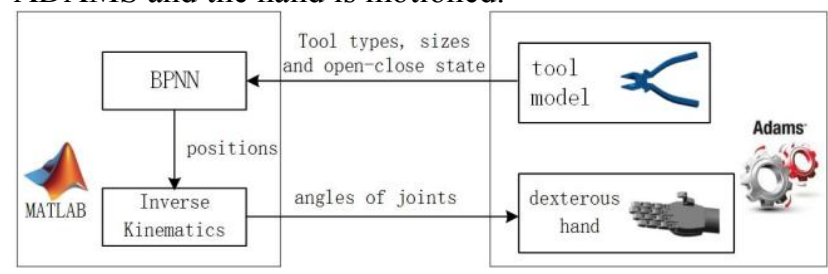

Fig.7. Dexterous hand operation simulation platform

\subsubsection{Tools in simple shape}

Three types of tools (long-cylinder, square, plate) are selected for the simulation experiments.

\section{A. Cylinder}

Select the cylindrical object to carry out the simulation experiment. The flashlight and the handle are tools in cylindrical shape.
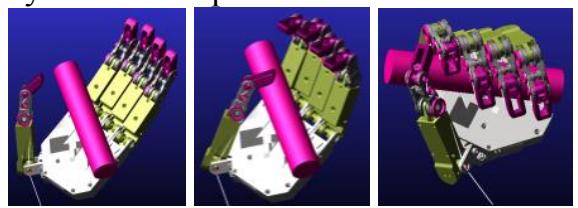

Fig.8. The grasping process of a cylindrical object

It can be seen from Fig.8 that when the dexterous hand is grasping the cylindrical object, the finger joints can be bent in order to reach the surface of the cylindrical object.

\section{B. Square}

Select the square object to carry out the simulation experiment. Square objects are common in experimental tools, such as boxes.
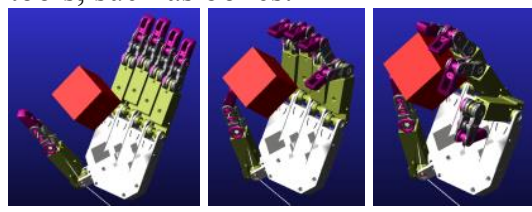

Fig.9. The grasping process of a square object

As can be seen from Fig.9, when grasping the target is as a square object, the fingers cannot make the knuckle close to the object as it can grasp a cylindrical object. Therefore, according to the gesture got from the BP neural network, the fingers bend to make sure the target is enveloped as much as possible.

\section{Plate objects}

Select the Board object to carry out the simulation experiment. In commonly used tools, the plate objects are often used as operating objects, such as planks, bricks, etc.
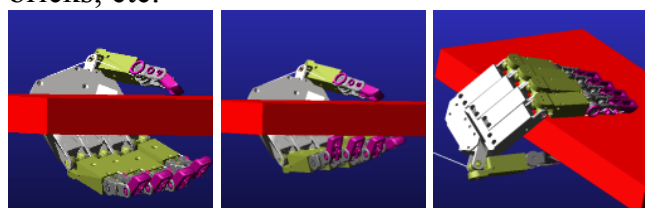

Fig.10. The grasping process of a plate object

As can be seen from Fig.10, the fingers cannot completely envelop the target in plate shape. In order to make the grasp more stable, the end knuckle should be as close as possible to the target.

\subsubsection{Tools in Complex shape}

Set up clamp in ADAMS, and select two different open and closed states.

Table 1 shows the size and the closed state of the tool. Table 2 shows the angles of finger joints after MATLAB calculation. Fig. 11 shows the operation motion of the dexterous hand. 
Table 1. Size and closed state of the clamp

\begin{tabular}{|l|l|c|}
\hline \multirow{2}{*}{ size } & $\mathrm{X}$ & $8.3 \mathrm{~mm}$ \\
\cline { 2 - 3 } & $\mathrm{Y}$ & $14.7 \mathrm{~mm}$ \\
\hline open angle & \multicolumn{2}{|c|}{$0.9^{\circ}$} \\
\hline
\end{tabular}

Table 2. Angles of finger joints

\begin{tabular}{|l|l|l|l|}
\hline Finger & joint $1\left(^{\circ}\right)$ & joint $2\left(^{\circ}\right)$ & joint $3\left(^{\circ}\right)$ \\
\hline 1 & 103.3 & 98.4 & 88.6 \\
\hline 2 & 25.1 & 68.9 & 100.2 \\
\hline 3 & 29.6 & 70.4 & 90.4 \\
\hline 4 & 15.1 & 69.8 & 80.2 \\
\hline 5 & 10.3 & 40.2 & 90.7 \\
\hline
\end{tabular}

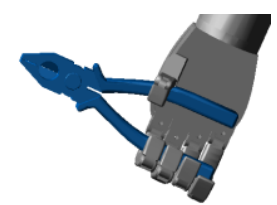

Fig.11. The dexterous hand operate a clamp in closed state

Table 3 shows the size and the open state of the tool. Table 4 shows the angles of finger joints after MATLAB calculation. Fig.12 shows the operation motion of the dexterous hand.

Tab.3. Size and closed state of the clamp

\begin{tabular}{|l|l|c|}
\hline \multirow{2}{*}{ size } & $\mathrm{X}$ & $14 \mathrm{~mm}$ \\
\cline { 2 - 3 } & $\mathrm{Y}$ & $14 \mathrm{~mm}$ \\
\hline open angle & \multicolumn{2}{|c|}{$21^{\circ}$} \\
\hline
\end{tabular}

Tab.4 Angles of finger joints

\begin{tabular}{|l|l|l|l|}
\hline Finger & joint $1\left(^{\circ}\right)$ & joint $2\left(^{\circ}\right)$ & joint $3\left(^{\circ}\right)$ \\
\hline 1 & 102.9 & 98.2 & 89.1 \\
\hline 2 & -5.2 & 29.8 & 90.4 \\
\hline 3 & 0.3 & 27.6 & 89.7 \\
\hline 4 & 5.4 & 0.1 & 88.9 \\
\hline 5 & 5.1 & 0.3 & 10.2 \\
\hline
\end{tabular}

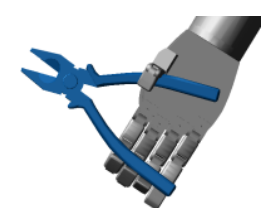

Fig.12. The dexterous hand operate a clamp in open state

It can be seen from the simulation results that motion planning method base on BPNN method is able to perform the motion task of planning finger joint angles and finally operate the tool.

\section{Conclusion}

The motion planning is an important issue in the robotic system. In this paper, BPNN was used to plan the rotation angles of each joint when the dexterous hand operate a tool combining with inverse kinematics of fingers. Finally, the simulation experiments verifies the effectiveness of this method. In the future, we tend to make a prototype of the dexterous hand and experiment this method on the hand of the machine.

\section{Acknowledgments}

This work was supported in part by the National Natural Science Foundation of China under Grant 61703067 and 51705058, Scientific and Technological Research Program of Chongqing Municipal Education Commission under Grant KJ1704072, Chongqing Research Program of Basic Research and Frontier Technology under Grant cstc2017jcyjAX0212.

\section{References}

1. M. T. Mason, J. K. Salisbury. Robot Hands and the Mechanics of Manipulation. MIT Press, Cambridge, USA, 1985: 3 93.

2. Lachnit H, Piepar W. Speed and accumcy effects of fingers and dexterity in 5-choice reaction tasks[J] Ergonomics, 1990, 33(12): 1443-1454.

3. Martin BJ, Armstrong TJ, Foulke JA, et d. Key - board reactical force and finger flexor electromyograms during OoInputer keyborad work[Jj. Human Factors, 1996, 38(4): 654664.

4. Dollar A M. The Highly Adaptive SDM Hand: Design and Performance Evaluation[J]. International Journal of Robotics Research, 2010, 29:585-597.

5. Cutkosky M R, Kao I. Computing and controlling compliance of a robotic hand[J]. Robotics \& Automation IEEE Transactions on, 1989, 5(2):151165.

6. Grebenstein M, Chalon M, Friedl W, et al. The hand of the DLR Hand Arm System: Designed for interaction[J]. The International Journal of Robotics Research, 2012, 31:1531-1555.

7. Lovchik C, Diftler M. The robonaut hand: a dexterous robot hand for space[C]//Proceedings of the IEEE International Conference on Automation and Robotics. Detroit, Michigan, 1999:907-912.

8. Bridgwater L B, Ihrke C A, Diftler M A 等. The Robonaut 2 hand - designed to do work with tools[C]. //Robotics and Automation, IEEE International Conference on. IEEE, 2012:3425 3430.

9. Rothling F, Haschke R, Steil J J 等. Platform portable anthropomorphic grasping with the bielefeld 20-DOF shadow and 9-DOF TUM hand[C]. //Intelligent Robots and Systems, 2007. IROS 2007. IEEE/RSJ International Conference on. IEEE, 2007:2951 - 2956.

10. Shadow Robot Company. Shadow Dextrous Hand Technical Specification [Z]. 2005,2. 